\title{
Co-Occurrence of Geriatric Syndromes and Diseases in the General Population: Assessment of the Dimensions of Aging
}

\author{
J.-F. Dartigues ${ }^{1}$, K. Le Bourdonnec ${ }^{1}$, M. Tabue-Teguo ${ }^{2}$, M. Le Goff', C. Helmer ${ }^{1}$, J.A.Avila-Funes ${ }^{3}$, G. Coureau ${ }^{1}$, \\ C. Feart ${ }^{l}$, K. Pérès ${ }^{1}$, R. Genuer ${ }^{l}$, L. Letenneur ${ }^{l}$, H. Amieva ${ }^{1}$, C. Proust-Lima ${ }^{1}$

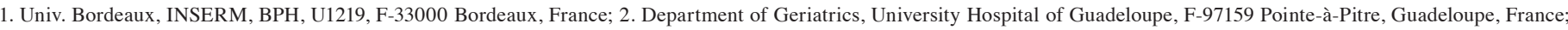 \\ 3. Department of Geriatrics. Instituto Nacional de Ciencias Médicas y Nutrición Salvador Zubirán, Mexico City, Mexico \\ Corresponding Author: Cécile Proust-Lima, Univ. Bordeaux, INSERM, BPH, U1219, F-33000 Bordeaux, France, cecile.proust-lima@inserm.fr
}

\begin{abstract}
OBJECTIVES: The co-occurrence of multiple medical or psychosocial conditions (geriatric syndromes (GS) and age-related diseases) is a growing concern in older people. Given the diversity of these conditions and their complex interactions, our aim was to determine whether they could be structured into synthetic dimensions in order to facilitate the management of multimorbidity.

DESIGN: The underlying structure of $10 \mathrm{GSs}$ and 8 age-related diseases was identified using a multiple correspondence analysis (MCA), and confronted to subjective and objective health outcomes.

SETTING: community residents from Bordeaux City (France) older than 75 years in 2010 .

PARTICIPANTS: 630 adults aged 75+ years who lived in Bordeaux and participated in the 10-year follow-up of the Three-City study.

MEASUREMENTS: GSs included physical frailty, cognitive impairment and dementia, dependency, depressive symptoms, polymedication, thinness, falls, sensory deficit, social isolation, incontinence. Age-related diseases were cancer, cardiac diseases, peripheral vascular diseases, diabetes, hypertension, pulmonary diseases, osteoporosis, other chronic diseases. Association of the MCA-derived independent dimensions was assessed with 10-year visit subjective health and well-being, and with incident death and entry into institution during the remaining cohort follow-up.

RESULTS: Most of the participants (82\%) had at least two age-related syndromes or diseases. The MCA structured the 18 conditions into three major dimensions: Degradation (D) driven by GS, Vascular (V), and Psychosocial (P) representing 68.7\%, 7.4\%, and 5.7\% of the total variance, respectively. Dimension D was a strong predictor of future death and institutionalization. Dimensions D and P were strongly associated with current well-being.

CONCLUSIONS: This work confirmed that multimorbidity is very common among older adults, and demonstrated the essential role of GS as manifestations of aging, even more than age-related diseases.
\end{abstract}

Key words: Geriatrics syndrome, age-related diseases, cohort study, death, institution.

\section{Introduction}

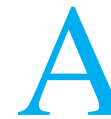
ging is characterized by the accumulation of agerelated physiological changes and diseases (1) but also changes in psycho-social functioning (2). The existence of multiple medical or psycho-social conditions in a single individual is a growing global health concern (3) and Received June 16, 2021 the available evidence on its causes, impact, and treatment is considered to be inadequate $(4,5)$. The current pandemic Coronavirus-Disease-19, particularly severe in older patients with multimorbidity or frailty (6), makes more obvious the need to better identify the epidemiological and pathological characteristics of the co-occurrence of aging disorders.

Given the diversity of indicators of age-related changes and their complex inter-dependencies, understanding the health status of older adults requires a precise description of these relationships and the identification of whether they can be structured into synthetic dimensions. The signification of these dimensions should be discussed on the basis of their relationship with objective and subjective health-related outcomes in older persons.

Numerous authors have proposed to define dimensions of aging that summarize the status of an individual. One or several dimensions have been highlighted. However, in previous papers, these dimensions of aging were most often a priori defined like for instance the frailty phenotype by Fried et al (7), the frailty index by Rockwood et al (8) for a unidimensional definition of frailty or the six dimensions considered by Pilotto (genetic, biological, functional, cognitive, psycho-social and socio-economic) for a multidimensional definition of frailty (9). An a priori definition could ignore important underlying latent dimensions that might explain some characteristics of aging such as the reduction of reserve capacities or a susceptibility to infections. An alternative consists in defining a posteriori dimensions of aging on the basis of the empirical analysis of associations between age-related changes.

Nonetheless, an essential prerequisite for the empirical study of health status of the older population is the representativeness of the studied sample. Any selection that could be related to a latent dimension itself should be avoided. This prevents the use of a clinical sample selected on the severity of a disease that justified the recourse to hospital setting; or on the opposite, healthy volunteers from the community likely selected by their good health status. Within the Bordeaux sample of the threeCity (3C Bordeaux) population-based cohort of adults aged 65 or older in 2000 (10), it was demonstrated that the 10-year visit sample was representative of community residents from Bordeaux City older than 75 years in 2010 (11). In this sample, we already conducted a first analysis of the co-occurrence of 
several geriatric syndromes. Using a multiple correspondence analysis (MCA) (12), we have shown that ten geriatric syndromes could be synthesized into two dimensions, one related to dementia, dependency and incontinence, and the other related to social isolation, depression and frailty. However, this work neither considered in the analysis specific age-related diseases like cancer or cardiovascular diseases nor explored the association of these dimensions with objective and subjective measures of health-related outcomes. A better understanding of the relationships and interactions between geriatric syndromes and specific diseases would allow the development of a more comprehensive and effective management of multimorbidity which is the norm in older patients.

In the present paper, we go one step further and leverage the representative $3 \mathrm{C}$ Bordeaux sample to (i) describe the underlying dimensions of 18 age-related indicators including geriatric syndromes and major chronic diseases occurring with aging; and (ii) evaluate the relationships between these dimensions and several outcomes of objective health (death, institutionalization), self-reported subjective health and wellbeing.

\section{Methods}

\section{Population Study}

The present research is an ancillary study of the 3C Study, a French prospective cohort study that aimed at evaluating the risk of dementia and cognitive impairment attributable to vascular risk factors. A detailed description of the $3 \mathrm{C}$ study has been provided elsewhere (10). Briefly, the total sample included 9,294 community-dwellers, aged 65 years or over, who were randomly selected between 1999 and 2000 from the electoral registries of three French cities: Bordeaux, Dijon, and Montpellier. The participants received home visits by a trained psychologist every two or three years up to the 17-year wave of follow-up. At entry and at each follow-up visit, the psychologists collected information during face-toface interviews and underwent a comprehensive evaluation of cognitive and physical function of the participant.

Following the previous analysis on the co-occurrence of geriatric syndromes in $3 \mathrm{C}$ (11), the study sample was limited to individuals living within the city of Bordeaux, who were seen at the ten-year follow-up in 2009 (thereafter called baseline). Among the 1104 individuals from the Bordeaux city enrolled in the 3C study in 1999-2000, 324 were dead by the 10-year visit (i.e., by the baseline for this study), 86 refused to participate, and 64 had moved away. The study sample thus comprised 630 participants seen at home by a psychologist and followed-up thereafter up to a maximum of 9.1 years.

The Ethical Committee of the University Hospital of Kremlin-Bicêtre (Paris, France) and Sud-Mediterranée 3 (Nimes, France) approved the 3C study, and all participants signed an informed consent.

\section{Variables assessment}

\section{Geriatric syndromes}

We focused on the same 10 Geriatric Syndromes (GS) as in our previous work (11) with a finer granularity for frailty and cognitive impairment.

Physical frailty: Five components were used to define physical frailty $(7,13)$ : weight loss/thinness (defined as a recent and unintentional weight loss of $\geq 3 \mathrm{~kg}$, or a body mass index $(\mathrm{BMI})<21 \mathrm{~kg} / \mathrm{m} 2$ ), exhaustion (defined as a positive response to at least one of the two questions from the Center for Epidemiological Studies Depression Scale (CES-D) (14): "I felt that everything I did was an effort" and "I could not get going"), slowness, weakness (both determined by scores in the lowest quintile on the timed 4-meter gait speed test adjusted for sex and height and on the grip strength test, respectively) and low physical activity (defined as not performing daily leisure activities such as walking, gardening or exercising at least once a week). Participants who met three or more criteria were classified as frail; those meeting one or two criteria, pre-frail; and those meeting none of the criteria, robust.

Dementia and mild cognitive impairment: At each follow-up visit, dementia diagnosis was based on a three-step procedure: dementia was pre-specified by the neuropsychologist who performed the neuropsychological evaluation at the participant's home; a clinical validation of the diagnosis was made at home by a neurologist or a geriatrician; a definitive diagnosis of dementia was ultimately made by a panel of independent neurologists based on the Diagnostic and Statistical Manual of Mental Disorders criteria (DSM-IV) (15). Nondemented participants were classified as having mild cognitive impairment (MCI) if they had a score lower than 17 at the total free recall score of the Free and Cued Selective Reminding Test (FCSRT) or lower than 40 at the total score of the FCSRT (16). In other cases, individuals were classified as cognitively normal.

Depressive symptoms were evaluated using the 20-item French version of the CES-D scale. Each item is scored from 0 to 3 depending on the frequency of occurrence in the past week, so that the total sum score ranges from 0 to 60 . Recommended cut-off scores of 17 and 23 were considered for significant depression in older men and older women, respectively (17).

Polymedication: At each visit, the participants were asked to present drug packages and prescription forms to assess all medications taken. Two groups of medication consumption were considered: no polymedication ( $<6$ drugs/day) and polymedication ( $\geq 6 \mathrm{drugs} /$ day) as recommended in previous studies (18).

Social Isolation: An individual was considered as socially isolated when he/she gave positive answers to both items "I live alone" and "I felt lonely" in the CES-D scale (19).

Thinness: Standardized measures of weight and size were recorded and used to calculate BMI. Participants with a BMI $<21 \mathrm{~kg} / \mathrm{m}^{2}$ were considered thin (20).

Falls: Participants were asked about the occurrence and number of falls since the previous follow-up visit, i.e., in the 
last 2 years on average (21)

Sensory deficits: An individual was considered sensory impaired when he/she reported either a vision trouble that is not corrected by lenses (self-reported or assessed at the visit) or a hearing trouble (positive response to "I have trouble following the conversation when two or more people talk at the same time or in a noisy background" or to "I have major hearing loss" to the question "Do you have hearing troubles?").

Dependency and incontinence: Participants were considered to be dependent if they were unable to perform at least one of the five following basic activities of daily living (ADL) without help (22): bathing, dressing, toileting, transferring from bed to chair and feeding. Incontinence (urinary and/or fecal incontinence) was considered as an impairment rather than a disability and was thus separated from the other ADL.

\section{Age-related diseases}

At each follow-up visit, participants were asked to report any new severe medical event or hospitalization since the last contact. We retained the 8 following age-related diseases.

Hypertension was defined by a systolic blood pressure (BP) $\geq 140 \mathrm{mmHg}$ and/or diastolic blood pressure $\geq 90 \mathrm{mmHg}$ using the mean of two measures and/or anti-hypertensive medication.

Diabetes: Participants were considered as diabetics when fasting glycemia was $\geq 7.0 \mathrm{mmol} / \mathrm{L}$ and/or they were taking antidiabetic treatment. They were classified as hyperglycemia if fasting glycemia was between 6.1 and $7 \mathrm{mmol} / \mathrm{L}$ without antidiabetic treatment.

Pulmonary disease was considered when the participants reported that they had lung disease or asthma.

Osteoporosis was self-reported.

Cardio-vascular diseases and stroke: When they were reported, all available clinical information were collected including emergency and hospitalization reports, neuroimaging reports (for stroke), and an interview with the patient's physician or family. All possible cardiovascular diseases and stroke events were adjudicated by two independent expert committees. Cardiac diseases were defined as a diagnosis of hospitalized angina, hospitalized myocardial infarction, or definite re-vascularization procedure. Stroke was defined according to the criteria of the World Health Organization, as a new focal neurological deficit of sudden or rapid onset, of presumed vascular origin, lasting for $24 \mathrm{~h}$ or more (23). Stroke and other self-reported peripheral vascular disease (e.g., aneurysm, angioplasty, arterial thrombosis) were classified as peripheral vascular disease.

Cancer: We merged data from the cancer registries of Gironde and the 3C Bordeaux cohort to identify older subjects with cancer in 2010 . We considered all malignant or benign tumors of the central nervous system as well as skin tumors (24). Cancer was classified as recent cancer if the diagnosis of cancer was made during the two years before the baseline screening or if the cancer was considered as progressive in 2010; it was classified as previous cancer if the diagnosis was made before 2008 and if the cancer was considered as nonprogressive.
Others: All other less frequent age-related morbidities reported by the participants were considered as a single category including chronic kidney disease, liver disease, HIV or Parkinson's disease.

\section{Health outcomes}

To address the secondary objective and evaluate the relationships between the dimensions of age-related indicators and several health-related outcomes, we considered four health outcomes: death, entry-in-institution, subjective health and well-being. Time to death and time to entry in institution were continuously collected up to the 17-year visit of the cohort corresponding to 7 years after this study baseline (and a maximum observed time of 9 years) where GS and agerelated morbidities were evaluated. Subjective health, assessed at baseline, was defined by the item "How do you feel about your health now?" with graded response from $1=$ Very Poor to 5=Excellent. Well-being, also collected at baseline, was defined as the sum-score of 4 reverse-scored items of the CES-D: "I felt I was just as good as other people", "I felt hopeful about the future", "I was happy", "I enjoyed life". Well-being score ranged from 0 (worse) to 12 (highest).

\section{Statistical analysis}

We used a MCA (12) to dissect the correlation structure of the $10 \mathrm{GS}$ and the 8 age-related diseases. MCA aims at retrieving the independent dimensions that explain most of the correlation within a set of categorical responses. It decomposes the inertia (i.e., measure of variance of the individual profiles around the average profile) into mutually independent dimensions. Each independent dimension is thus characterized by the percentage of the total inertia it explains. As crude values tend to heavily underestimate the actual percentages of inertia in MCA, actual percentages were computed using the Greenacre correction (25). To ensure a meaningful description and interpretation of the information, we statistically selected the dimensions that explain the highest percentages of total inertia using the Kaiser criterion (i.e., dimensions with eigen values above $1 / \mathrm{k}$, $\mathrm{k}$ being the number of variables).

MCA provides in the end a graphical tool to retrieve the most important mutually independent dimensions and their constituents. On each dimension, the distance between categorical responses translates their level of association: two categories shared by about the same individuals will be very close while categories possessed by quite distinct sets of individuals will be opposite on the dimension. Categories around zero will not be part of the dimension constituents.

To account for missing responses without adding a missing category which is difficult to interpret (11), we used a regularized iterative MCA in which missing responses are internally imputed under missing at random mechanism (26). We also regrouped the rare responses to avoid the overinfluence of rare modalities in MCA.

Once the structure in independent dimensions established, we proceeded to posterior analyses. First, additional variables 
Table 1. Characteristics of the sample of 630 participants living in Bordeaux City Area within the 3C study at baseline (2009) and over time for entry in institution and death incidence

\begin{tabular}{|c|c|c|c|c|c|c|}
\hline Variables & $\begin{array}{c}\text { Overall } \\
(\mathrm{N}=630) \\
\mathbf{n}(\%)\end{array}$ & NA & $\begin{array}{c}\text { Women } \\
(\mathrm{N}=437(69.4 \%)) \\
\text { n }(\%)\end{array}$ & NA & $\begin{array}{c}\text { Men } \\
(\mathrm{N}=193(30.6 \%)) \\
n(\%)\end{array}$ & NA \\
\hline \multicolumn{7}{|l|}{ Age } \\
\hline$[75 ; 80[$ & $181(29)$ & & $120(27)$ & & $61(31)$ & \\
\hline$[80 ; 85[$ & $216(34)$ & & $147(34)$ & & $69(36)$ & \\
\hline$[85 ;+[$ & $233(37)$ & & $170(39)$ & & $63(33)$ & \\
\hline \multicolumn{7}{|l|}{ Educational level } \\
\hline Lower than high school & $238(38)$ & & $189(43)$ & & $49(25)$ & \\
\hline High school and higher & $392(62)$ & & $248(57)$ & & $144(75)$ & \\
\hline \multicolumn{7}{|l|}{ Geriatric Syndromes } \\
\hline \multicolumn{7}{|l|}{ Frailty } \\
\hline Pre-frailty & $150(24)$ & & $104(24)$ & & $46(24)$ & \\
\hline Frailty & $208(33)$ & & $161(37)$ & & $47(24)$ & \\
\hline \multicolumn{7}{|l|}{ Dementia } \\
\hline MCI & $181(29)$ & & $122(28)$ & & $59(31)$ & \\
\hline Dementia & $98(16)$ & & $71(16)$ & & $27(14)$ & \\
\hline Depression & $76(13)$ & 59 & $62(16)$ & 41 & $14(8)$ & 18 \\
\hline Polymedication & $319(51)$ & & $230(53)$ & & $89(46)$ & \\
\hline Isolation & $53(9)$ & 30 & $48(12)$ & 25 & $5(3)$ & 5 \\
\hline Thinness & $51(8)$ & & $43(10)$ & & $8(4)$ & \\
\hline Falls & $266(42)$ & & $202(46)$ & & $64(33)$ & \\
\hline Sensory deficit & $82(13)$ & & $48(11)$ & & $34(18)$ & \\
\hline Dependency to ADL & $62(10)$ & & $47(11)$ & & $15(8)$ & \\
\hline Incontinence & $80(13)$ & & $66(15)$ & & $14(7)$ & \\
\hline \multicolumn{7}{|l|}{ Age-related diseases } \\
\hline Hypertension & $441(72)$ & 15 & $306(72)$ & 11 & $135(72)$ & 4 \\
\hline \multicolumn{7}{|l|}{ Diabetes } \\
\hline Hyperglycemia & $26(4)$ & & $14(3)$ & & $12(6)$ & \\
\hline Diabetes & $83(13)$ & & $49(11)$ & & $34(18)$ & \\
\hline Pulmonary disease & $160(25)$ & & $113(26)$ & & $56(29)$ & \\
\hline Osteoporosis & $103(17)$ & 29 & $100(24)$ & 29 & $3(2)$ & \\
\hline Cardiac disease & $56(9)$ & 13 & $39(9)$ & 10 & $17(9)$ & 3 \\
\hline Vascular disease & $48(7)$ & & $25(6)$ & & $23(12)$ & \\
\hline Cancer & & 5 & & 5 & & \\
\hline Previous cancer & $45(7)$ & & $25(6)$ & & $20(10)$ & \\
\hline Recent cancer & $28(4)$ & & $16(4)$ & & $12(6)$ & \\
\hline Other severe disease & $23(4)$ & & $13(3)$ & & $10(5)$ & \\
\hline \multicolumn{7}{|l|}{ Health Outcomes } \\
\hline Incident death & $288(46)$ & & $183(42)$ & & $105(54)$ & \\
\hline Survival Time in years (median) & 8.39 & & 8.39 & & 6.94 & \\
\hline Participants living in institution at baseline & $53(8)$ & & $42(10)$ & & $11(6)$ & \\
\hline Incident entries in institution & $75(13)$ & & $59(15)$ & & $16(9)$ & \\
\hline Subjective health & 26 & & 19 & & 7 & \\
\hline Very poor & $9(2)$ & & $6(1)$ & & $3(2)$ & \\
\hline Poor & $51(8)$ & & $38(9)$ & & $13(7)$ & \\
\hline Fair & $232(38)$ & & $163(39)$ & & $69(37)$ & \\
\hline Good & $272(45)$ & & $184(44)$ & & $88(47)$ & \\
\hline Excellent & $40(7)$ & & $27(7)$ & & $13(7)$ & \\
\hline Wellbeing score (mean (sd)) & $8.60(3.00)$ & 82 & $8.29(3.13)$ & 61 & $9.28(2.58)$ & 21 \\
\hline
\end{tabular}

Note. MCI = Mild Cognitive impairment $\mathrm{ADL}=$ Activity of Daily Life; NA = Not Available (missing) information

were projected onto the structure to illustrate the dimensions. Second, the association of the identified dimensions with each health outcomes was separately assessed either using Cox models for time-to-death and time-to-entry-in-institution, and proportional odds logistic regression for graded responses to subjective health and well-being. Regressions were systematically adjusted for sex, education and age at baseline (with a nonlinear relationship approximated by cubic splines). A risk of 5\% was chosen for statistical significance, and the $95 \%$ confidence intervals $(95 \% \mathrm{CI})$ were provided. Analyses were 
Table 2. Contribution (reported in \%) of the 40 health characteristics (from 10 Geriatric Syndromes and 8 chronic diseases) to the three main dimensions (D, V and P) identified in MCA at the 10-year follow-up of the 3C study ( $\mathrm{n}=630$ ). Are reported in Bold the main contributors of each dimension

\begin{tabular}{|c|c|c|c|}
\hline Variable Response & 1st dimension Degradation (D) & 2nd dimension Vascular (V) & 3rd dimension Psycho-Social (P) \\
\hline \multicolumn{4}{|l|}{ Geriatric Syndromes } \\
\hline Frailty & 12.34 & 0.45 & 1.19 \\
\hline Pre-frailty & 0.64 & 0.71 & 0.48 \\
\hline Robustness & 6.15 & $<0.01$ & 0.19 \\
\hline Dementia & 11.52 & 0.21 & 9.92 \\
\hline MCI & 0.01 & 1.12 & 2.30 \\
\hline Normal Cognition & 3.40 & 1.00 & 0.33 \\
\hline Depression & 6.00 & 1.67 & 9.50 \\
\hline No depression & 1.02 & 0.28 & 1.61 \\
\hline Polymedication & 3.68 & 5.56 & 2.59 \\
\hline No polymedication & 3.78 & 5.71 & 2.65 \\
\hline Isolation & 0.52 & 5.34 & 20.61 \\
\hline No isolation & 0.05 & 0.53 & 2.05 \\
\hline Thinness & 0.62 & 11.66 & 1.73 \\
\hline No thinness & 0.05 & 1.03 & 0.15 \\
\hline Falls & 2.03 & 0.11 & 0.33 \\
\hline No falls & 1.48 & 0.08 & 0.03 \\
\hline Sensory deficit & 2.73 & 1.37 & 0.23 \\
\hline No sensory deficit & 0.41 & 0.21 & 0.03 \\
\hline Dependency to ADL & 16.06 & 0.16 & 9.97 \\
\hline No dependency to ADL & 1.75 & 0.02 & 1.09 \\
\hline Incontinence & 11.35 & 0.32 & 3.92 \\
\hline No incontinence & 1.65 & 0.05 & 0.57 \\
\hline \multicolumn{4}{|l|}{ Age-related diseases } \\
\hline Hypertension & 0.09 & 5.69 & 0.75 \\
\hline No hypertension & 0.23 & 14.37 & 1.90 \\
\hline Diabetes & 0.29 & 13.14 & 1.74 \\
\hline Hyperglycemia & 0.29 & 0.86 & 0.08 \\
\hline No diabetes & 0.01 & 2.73 & 0.35 \\
\hline Pulmonary disease & 0.82 & 0.01 & 4.91 \\
\hline No pulmonary disease & 0.30 & 0.01 & 1.80 \\
\hline Osteoporosis & 1.41 & 5.70 & 7.38 \\
\hline No osteoporosis & 0.30 & 1.22 & 1.59 \\
\hline Cardiac & 3.52 & 6.67 & 0.03 \\
\hline No cardiac & 0.36 & 0.68 & $<0.01$ \\
\hline Vascular disease & 1.23 & 7.01 & 2.30 \\
\hline No vascular disease & 0.10 & 0.58 & 0.19 \\
\hline Recent cancer & 0.09 & 1.91 & 0.73 \\
\hline Previous cancer & 0.01 & 0.49 & 2.26 \\
\hline No cancer & 0.01 & 0.26 & 0.06 \\
\hline Other severe disease & 3.59 & $<0.01$ & 2.26 \\
\hline No other severe disease & 0.14 & $<0.01$ & 0.11 \\
\hline
\end{tabular}


performed using $\mathrm{R}$, version 3.6.3 and $\mathrm{R}$ package FactoMineR (27).

\section{Results}

Among the 630 participants older than 75 years (437 women and 193 men, mean age 83.3), the most frequent GS or agerelated diseases were hypertension $(72 \%)$, followed by frailty or pre-frailty $(57 \%)$, polymedication $(51 \%)$, cognitive impairment or dementia (45\%) and falls (42\%) (Table 1). A total of 514 participants $(82 \%)$ had at least two age-related syndromes or diseases and could be considered as having multimorbidity (329 with at least two GS, 308 with at least two age-related diseases). The MCA was performed on the 40 categories stemming from the 18 variables with at least $5 \%$ occurrence. Three dimensions were selected with a percentage of the global inertia higher than the Kaiser criterion threshold of $5.5 \%(=1 / 18)$. After Greenacre correction, the first three principal dimensions explained $81.2 \%$ of the total variance with $68.7 \%, 7.4 \%$ and $5.7 \%$ for the first, second and third dimension, respectively. The contributions of the 40 health responses to the three first dimensions are presented in Table 2, and the corresponding mappings are displayed in Figure 1. The main constituents of the first dimension were physical frailty, dementia, dependency, and incontinence for the positive side (all with contributions above $11.4 \%$ ), and robustness for the negative side (with contribution of $6.2 \%$ ). No chronic disease was clearly related to this dimension. According to its main constituents, we called this dimension Degradation (D). The second dimension was primarily characterized by diabetes for positive side and absence of hypertension and thinness for the negative side, all with contributions higher than $11.7 \%$ each. Also substantially contributed to the second axis (around 6-7\% each) vascular disease, cardiac disease and presence of polymedication for the positive side, and osteoporosis and absence of polymedication for the negative side (Figure 1 top panel). We called the second dimension Vascular (V). Finally, the main contributors of the third dimension were isolation $(20.6 \%)$, depression $(9.5 \%)$ and osteoporosis (7.4\%) on the positive side, and dementia (9.9\%) and ADL dependency $(10.0 \%)$ on the negative side (Figure 1 bottom panel). We called the third dimension Psychosocial (P).

Age was strongly and almost linearly related to D (Figure 2 top panel) while sex was related to $\mathrm{P}$, women being on the upper part of the axis driven by isolation, depression and osteoporosis and men in the opposite part of the axis (Figure 2 bottom panel). Educational level (high school and higher versus lower than high school) was not clearly related to any of the three dimensions.

\section{Association with health outcomes}

During the subsequent follow-up, 288 participants died with a median time to death of 8.39 years. Adjusted for age, sex and education, D was associated with the risk of dying with a Hazard Ratio (HR) of 1.87 [95\%CI=(1.67,2.09)] for an increase in the dimension of one standard deviation (SD) $(1 \mathrm{SD}=0.39$ in the scale displayed in Figure 1) (Table 3). For comparison, the HR related to an increase in age from 80 to 83 and from 83 to 86 were $1.23[95 \% \mathrm{CI}=(1.03,1.47))$ and $1.50(95 \% \mathrm{CI}=(1.23,1.85)]$, respectively. $\mathrm{V}$ and $\mathrm{P}$ were much less associated with the risk of death, with slightly higher risks of death for higher $\mathrm{V}$ and lower $\mathrm{P} \quad[\mathrm{HR}=1.16(1.03,1.30)$ and $\mathrm{HR}=0.91(0.82,1.02)$ for one $\mathrm{SD}$ change of $\mathrm{V}$ and $\mathrm{P}$, respectively; $1 \mathrm{SD}=0.3$ in the original scales displayed in Figure $1]$.

Figure 1. Mapping of the 40 health characteristics (from 10 Geriatric Syndromes and 8 chronic diseases) on the three main dimensions identified in MCA at the 10-year follow-up of the 3 C study $(n=630)$
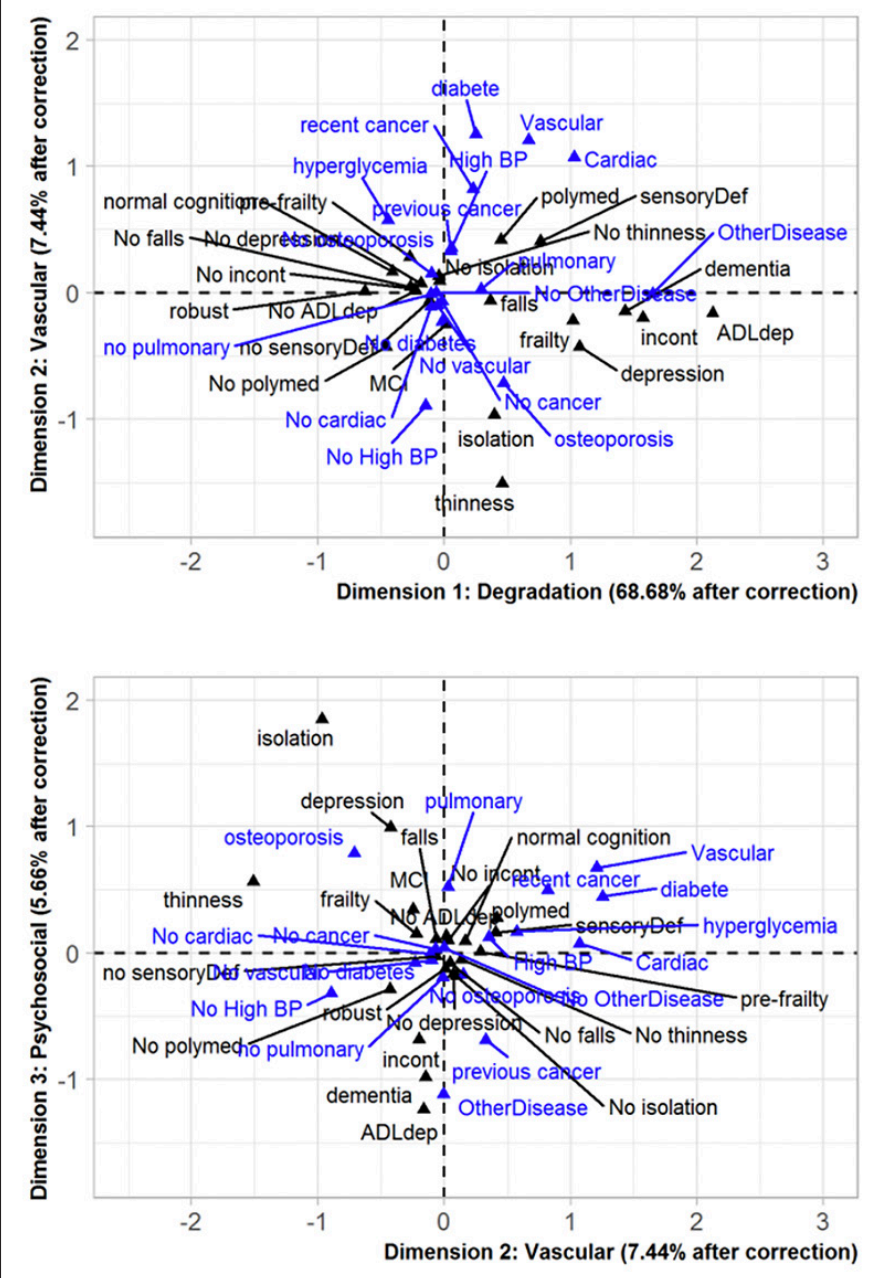

Top: 2-D mapping with first dimension versus second dimension; Bottom: 2-D mapping with second dimension versus third dimension.

Among the 577 participants living at home at this study baseline (53 were already in institution), 75 (13\%) entered in institution during the subsequent follow-up. Adjusted for age, sex and education, higher location on $\mathrm{D}$ was associated with a higher risk of institutionalization $(\mathrm{HR}=1.56(1.23,1.98)$ for one SD increase). The other dimensions were not significantly associated with institutionalization ( $\mathrm{p}>0.232)$.

Finally, half of the subjects reported a good or excellent subjective health at baseline $(10 \%$ reported a poor or very poor subjective health) with no difference according to sex, and the average score of wellbeing was $8.6(\mathrm{SD}=3)$ with 
women reporting a 1-point lower score than men in average. Adjusted for age, education and sex, D and $\mathrm{P}$ were both strongly associated with degraded subjective health [OddsRatio $(\mathrm{OR})=0.45(0.37,0.54)$ and $\mathrm{OR}=0.58(0.49,0.68)]$ per unit increase in subjective health for a one SD increase in $\mathrm{D}$ and $\mathrm{P}$, respectively) and lower well-being $[\mathrm{OR}=0.44(0.35,0.55)$ and $\mathrm{OR}=0.59(0.49,0.69)]$ per unit increase in wellbeing for a one SD increase in $\mathrm{D}$ and $\mathrm{P}$, respectively). In contrast, $\mathrm{V}$ was poorly associated with degraded subjective health [OR=0.77 $(0.66,1.08)$ per unit increase in subjective health for a one SD increase in V] but subjects with higher scores to $\mathrm{V}$ simultaneously reported better scores of well-being [OR $=1.43$ $(1.23,1.68)$ per unit increase of well-being for one SD increase in $\mathrm{V}]$.

Figure 2. Mapping of the 7 illustrative characteristics (age in 3 categories, sex and education) on the three main dimensions identified in MCA at the 10-year follow-up of the $3 \mathrm{C}$ study $(n=630)$

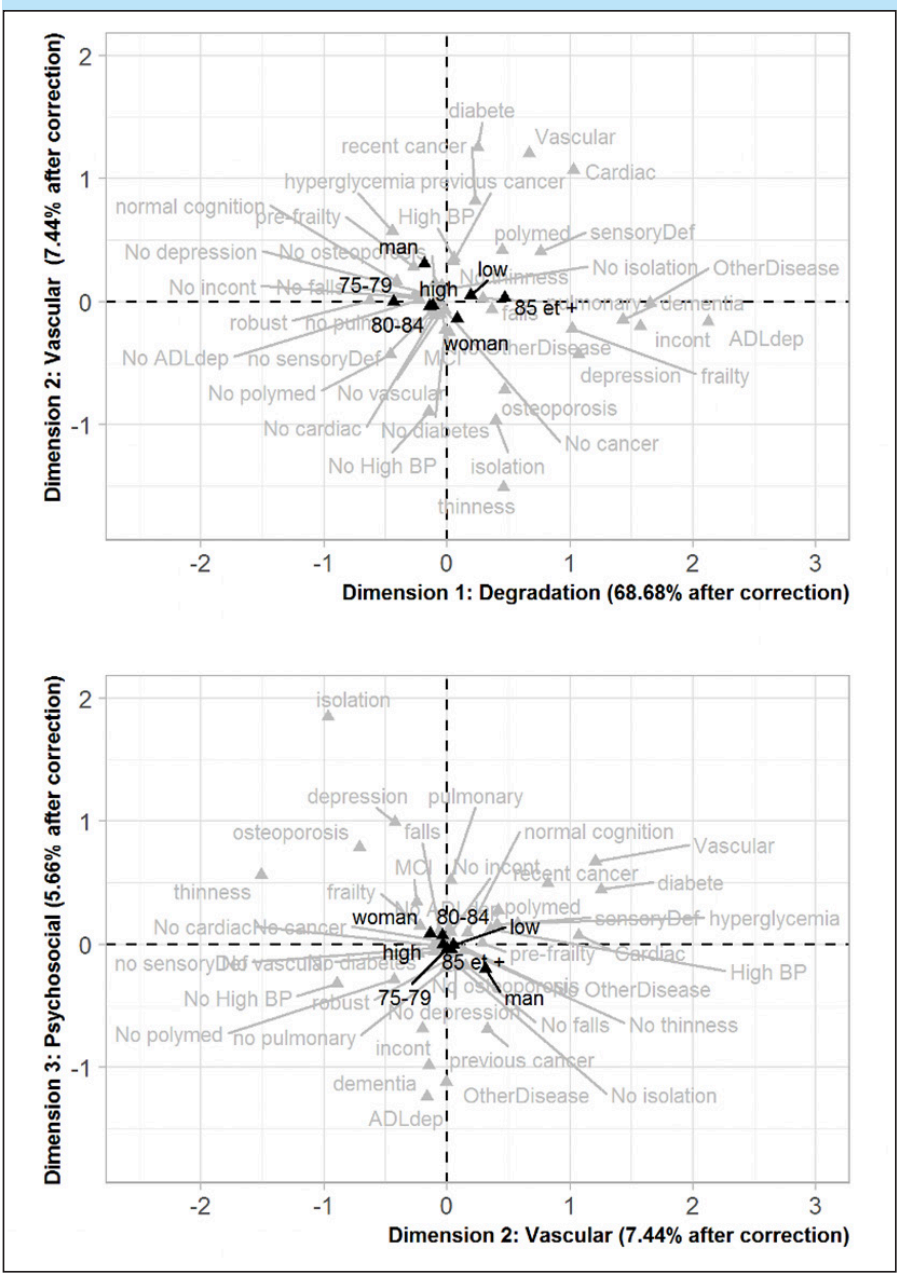

Top: 2-D mapping with first dimension versus second dimension; Bottom: 2-D mapping with second dimension versus third dimension.

\section{Discussion}

By analyzing 40 characteristics of aging stemmed from 18 GS and diseases, our study confirmed the major prevalence of multimorbidity in the population of $75+$ years, and the necessity to better understand this phenomenon for the management of older patients (28). Eighty two percent of older adults reported at least two diseases or GS accordingly with available literature (5). Using MCA, we synthesized the multiple and complex interactions between diseases and GS into three independent dimensions. The first dimension (D for Degradation), essentially related to GS (Dependency, Dementia, Frailty, Incontinence) was by far the most important ( $68.7 \%$ of total inertia) and represents the global function. Although closely related to age, it added critical information for the prediction of health-related outcomes (death, entry into institution, subjective health, well-being). Interestingly, this dimension was also substantially defined by the absence of frailty or pre-frailty in the negative direction. This underlines that positive parameters of health are as important as negative parameters in the prediction of health-related outcomes, and thus calls for promotion of the emerging successful aging (29). By opposing global degradation to robustness, this first dimension $\mathrm{D}$ could be considered as a good reflection of the real degradation of the health with aging, but also as a good estimate of reserve capacities.

The second (V for Vascular) and third ( $\mathrm{P}$ for Psychosocial) dimensions explained much less inertia in this population $(7.4 \%$ and $5.7 \%$, respectively) but emphasized additional relevant continuums. V driven by age-related diseases, opposed cardiovascular diseases and risk factors (diabetes, cardiac disease, peripheral vascular diseases) to the absence of hypertension, the absence of polymedication, and thinness. This dimension relying on cardio-vascular diseases, was not as predictive of health outcomes as D. It was only associated with a slightly increased risk of death and a better wellbeing. This suggests that these chronic conditions do not have a significant impact on death and other health outcomes in a population as old as ours by comparison with non-vascular chronic diseases, more related to degenerative mechanism like dementia.

Interestingly, dimension $\mathrm{P}$ was also mostly driven by GS. It opposed psycho-social to organic aspects of aging with depression, isolation on the positive side, and dementia and dependency on the negative side. Only one chronic disease was related to the dimension, osteoporosis on the positive side. P, closely associated with sex, substantially complements $\mathrm{D}$ for prediction of subjective health-related outcomes with a higher risk of poor subjective health and well-being. This result highlights the importance of the management of isolation for the well-being of older adults in the general population.

Some frequent chronic diseases are weakly related to the dimensions obtained with the MCA, and particularly cancer and cardiac diseases. This was somewhat expected since the GS are partially the consequences of the diseases that occur before GS in the causal chain that leads from the normal state to death. These results do not mean that these chronic diseases are not predictive of death or other health outcomes; this would be in contradiction with the scientific literature (5). It means that if chronic diseases are predictive of health-related outcomes, it is essentially through their consequences, GS (30, 31). This finding could explain the failure of comprehensive gerontological management trials that are much more focused on chronic diseases than GS (32). Moreover, another 
Table 3. Association between the three independent dimensions D, V and P (for Degradation, Vascular and Psychosocial, respectively) and health outcomes: incidence of death, entry in institution, subjective health and wellbeing

\begin{tabular}{|c|c|c|c|c|c|c|c|c|}
\hline & \multicolumn{2}{|l|}{ Death } & \multicolumn{2}{|c|}{ Institution } & \multicolumn{2}{|c|}{ Subjective health } & \multicolumn{2}{|l|}{ Wellbeing } \\
\hline & HR $(95 \%$ CI $)$ & $\mathbf{p}$ & HR (95 \% CI) & $\mathbf{p}$ & OR $(95 \% \mathrm{CI})$ & $\mathbf{p}$ & OR $(95 \% \mathrm{CI})$ & $\mathbf{p}$ \\
\hline Dimension $\mathrm{D}$ (for one SD change) & $1.87[1.67 ; 2.09]$ & $<0.001$ & $1.56[1.23 ; 1.98]$ & $<0.001$ & $0.45[0.37 ; 0.54]$ & $<0.001$ & $0.44[0.35 ; 0.55]$ & $<0.001$ \\
\hline Dimension $\mathrm{P}$ (for one SD change) & $0.91[0.82 ; 1.02]$ & 0.116 & $0.92[0.75 ; 1.19]$ & 0.636 & $0.58[0.49 ; 0.68]$ & $<0.001$ & $0.59[0.49 ; 0.69]$ & $<0.001$ \\
\hline Age (77 yo) & $0.61[0.50 ; 0.74]$ & & $0.19[0.08 ; 0.44]$ & & $1.04[0.65 ; 1.67]$ & 0.874 & $0.80[0.51 ; 1.26]$ & 0.334 \\
\hline Age (86 yo) & $1.50[1.23 ; 1.85]$ & & $1.47[0.95 ; 2.28]$ & & $1.09[0.79 ; 1.51]$ & 0.596 & $0.90[0.64 ; 1.26]$ & 0.541 \\
\hline Age (90 yo) & $2.54[1.83 ; 3.52]$ & & $2.09[1.23 ; 3.57]$ & & $1.28[0.76 ; 2.16]$ & 0.347 & $0.90[0.54 ; 1.50]$ & 0.699 \\
\hline Woman (vs man) & $0.51[0.39 ; 0.66]$ & $<0.001$ & $1.71[0.92 ; 3.29]$ & 0.086 & $1.19[0.83 ; 1.69]$ & 0.338 & $0.78[0.56 ; 1.10]$ & 0.161 \\
\hline High (vs low) educational level & $1.01[0.79 ; 1.28]$ & 0.953 & $1.95[1.02 ; 2.88]$ & 0.041 & $1.30[0.94 ; 1.80]$ & 0.118 & $0.76[0.55 ; 1.06]$ & 0.077 \\
\hline
\end{tabular}

Note. HR=hazard ratio; OR = odds ratio for one category increase; SD = standard deviation; nonlinear association with age were obtained with cubic splines reported for the $10 \%, 30 \%$, $50 \%, 70 \%$ and $90 \%$ of the age distribution.

consequence is that a disease occurring in an older adult with good reserve capacities, for instance without criteria of physical frailty, has certainly a better prognosis. This is effectively the case for many diseases and even the Covid-19 (6).

Faced with the complexity and multi-dimensionality of aging and the difficulties of its management, new approaches are emerging with the idea of promoting a holistic vision of aging rather than the specific management of each symptom. This conception is consistent with the general hypothesis of gerosciences that posits that manipulation of aging will delay the occurrence of many chronic diseases (4), or with multidimensional approaches of frailty (9). Our analysis is in line with these works, and further illustrates in a general population of $75+$ the existence of multiple dimensions of aging, namely the global degradation, the cardio-vascular morbidity and the isolation, and among them the broad predominance of global degradation strongly related to healthrelated outcomes, which could be considered as a general clinical manifestation of biological aging (4).

A major strength of this work lies in the high quality of the data. First, it was previously demonstrated that the sample reliably represented the community residents from Bordeaux City older than 75 years in 2010 (11), such representiveness is a requirement for the generazibility of the findings. Second, as stemmed from a cohort study dedicated to cerebral and vascular aging, the data collection was very thorough particularly for complex key manifestations such as MCI, dementia, frailty, dependency which are particularly difficult to diagnose, or cancer and cardiac diseases. Another strength lies in the chosen statistical methodology. Interactions between diseases and GS in older adults are certainly the major impediment to the understanding and management of health problems in older adults (32). MCA is a methodology specifically designed to address such issue; it aims at disentangling interactions between manifestations and synthesizing them into several independent dimensions (33), that may further be challenged with healthrelated outcomes to understand their signification and validate their usefulness for the management of older adults. MCA was preferred over clustering approaches as our aim was to identify the underlying dimensions of GSs and age-related diseases rather than classify individuals into subgroups of multimorbidity (34). This work also has weaknesses. They essentially relate to the relatively limited sample size, the absence of data for some chronic diseases like osteoarticular diseases, and the absence of biomarkers for Alzheimer's Disease diagnosis.

To conclude, this work confirmed the essential role of GS as manifestations of aging, even more than age-related diseases, but also underlined the importance of the absence of frailty that translates the reserve capacities, the robustness of the patient, and opens to the consideration of successful aging as a critical element in the management of older adults along with multimorbidity.

\section{Conflict of Interest: none.} 2012)

Funding: This work was supported by the Plan National Alzheimer France (2008-

Authors contributions: All the authors contributed significantly to the work at different stages: conception and design (all the authors), acquisition of data (Catherine Helmer, Mélanie Le Goff, Jean-François Dartigues), analysis (Kateline Le Bourdonnec, Robin Genuer, Cécile Proust-Lima), interpretation (all authors), manuscript draft (Jean-François Dartigues, Cécile Proust-Lima), critical revision (all authors). In addition, all the authors gave their final approval of the version to be submitted. Finally, we confirm that this manuscript has never been published and is not being simultaneously considered for publication elsewhere.

Ethical standards: This work complies with the French current laws.

\section{References}

1. Geokas MC, Lakatta EG, Makinodan T, Timiras PS. The aging process. Ann Intern Med 1990;113:455-66.

2. Wister A, Lear S, Schuurman N, MacKey D, Mitchell B, Cosco T, Fyffe I. Development and validation of a multi-domain multimorbidity resilience index for an older population: results from the baseline Canadian Longitudinal Study on Aging. BMC Geriatr 2018;18:170.

3. World Health Organization, 2019. Integrated care for older people (ICOPE): guidance for person-centred assessment and pathways in primary care. Available at: https://apps. who.int/iris/handle/10665/326843.

4. Ferrucci L, Gonzalez-Freire M, Fabbri E, Simonsick E, Tanaka T, Moore Z, Salimi S, Sierra F, de Cabo R. Measuring biological aging in humans: A quest. Aging Cell 2020;19:e13080.

5. Marengoni A, Angleman S, Melis R, Mangialasche F, Karp A, Garmen A, Meinow B, Fratiglioni L. Aging with multimorbidity: a systematic review of the literature. Ageing 
Res Rev 2011;10:430-9.

6. Hewitt J, Carter B, Vilches-Moraga A, et al. The effect of frailty on survival in patients with COVID-19 (COPE): a multicentre, European, observational cohort study. Lancet Public Health 2020;5:e444-e451.

7. Fried LP, Tangen CM, Walston J, Newman AB, Hirsch C, Gottdiener J, Seeman T, Tracy R, Kop WJ, Burke G, McBurnie MA. Frailty in older adults: evidence for a phenotype. J Gerontol A Biol Sci Med Sci 2001;56:M146-56.

8. Rockwood K, Mitnitski A. Frailty in relation to the accumulation of deficits. J Gerontol A Biol Sci Med Sci 2007;62:722-7.

9. Pilotto A, Custodero C, Maggi S, Polidori MC, Veronese N, Ferrucci L. A multidimensional approach to frailty in older people. Ageing Research Reviews 2020;60:101047.

10. 3C Study Group. Vascular factors and risk of dementia: design of the Three-City Study and baseline characteristics of the study population. Neuroepidemiology 2003;22:316 325 .

11. Tabue-Teguo M, Grasset L, Avila-Funes JA, Genuer R, Proust-Lima C, Péres K, Féart C, Amieva H, Harmand MG-C, Helmer C, Salles N, Rainfray M, Dartigues JF. Prevalence and Co-Occurrence of Geriatric Syndromes in People Aged 75 Years and Older in France: Results From the Bordeaux Three-city Study. J Gerontol A Biol Sci Med Sci 2017;73:109-116.

12. Benzecri. Correspondence Analysis Handbook. New York, CRC Press, 1992.

13. Avila-Funes JA, Pelletier A, Meillon C, Catheline G, Periot O, Trevin O-Frenk I, Gonzalez-Colaço M, Dartigues J-F, Pérès K, Allard M, Dilharreguy B, Amieva H. Vascular Cerebral Damage in Frail Older Adults: The AMImage Study. J Gerontol A Biol Sci Med Sci 2017;72:971-977.

14. Radloff LS. The CES-D Scale: A Self-Report Depression Scale for Research in the General Population. Applied Psychological Measurement 19771:385-401

15. American Psychiatric Association. Diagnostic and Statistical Manual of Menta Disorders. Fifth Edition. Arlington (VA), American Psychiatric Association doi:10.1176/appi.books.9780890425596.

16. Sarazin M, Berr C, De Rotrou J, Fabrigoule C, Pasquier F, Legrain S, Michel B Puel M, Volteau M, Touchon J, Verny M, Dubois B. Amnestic syndrome of the medial temporal type identifies prodromal AD: a longitudinal study. Neurology 2007;69:1859-1867.

17. Antonucci TC, Fuhrer R, Dartigues JF. Social relations and depressive symptomatology in a sample of community-dwelling French older adults. Psychol Aging 1997;12:189-95.

18. Jyrkka J, Enlund H, Korhonen MJ, Sulkava R, Hartikainen S. Polypharmacy status as an indicator of mortality in an elderly population. Drugs Aging 2009;26:1039-48.

19. Tabue Teguo M, Simo-Tabue N, Stoykova R, Meillon C, Cogne M, Amieva H, Dartigues JF. Feelings of Loneliness and Living Alone as Predictors of Mortality in the Elderly: The PAQUID Study. Psychosom Med 2016;78:904-909.

20. Guigoz Y, Vellas B, Garry PJ. Assessing the nutritional status of the elderly: The Mini Nutritional Assessment as part of the geriatric evaluation. Nutr Rev 1996;54:S59-65.
21. Berdot S, Bertrand M, Dartigues JF, Fourrier A, Tavernier B, Ritchie K, Alperovitch A. Inappropriate medication use and risk of falls--a prospective study in a large community-dwelling elderly cohort. BMC Geriatr 2009;9:30.

22. Katz S, Ford AB, Moskowitz RW, Jackson BA, Jaffe MW. Studies of Illness in the Aged: The Index of ADL: A Standardized Measure of Biological and Psychosocial Function. JAMA 1963;185:914-919.

23. Samieri C, Perier M-C, Gaye B, Proust-Lima C, Helmer C, Dartigues J-F, Berr C, Tzourio C, Empana J-P. Association of Cardiovascular Health Level in Older Age With Cognitive Decline and Incident Dementia. JAMA 2018;320:657-664.

24. Galvin A, Helmer C, Coureau G, Amadeo B, Rainfray M, Soubeyran P, Dartigues J-F, Pérès K, Bellera C, Delva F, Mathoulin-Pélissier S. Determinants of functional decline in older adults experiencing cancer (the INCAPAC study). J Geriatr Oncol 2019;10:913-920.

25. Abdi H, valentin D. Multiple correspondence analysis. Encyclopedia of Measurement and Statistics, CA, Sage, 2007;pp 651-657. Thousand Oaks.

26. Josse J, Chavent M, Liquet B, Husson F. Handling Missing Values with Regularized Iterative Multiple Correspondence Analysis. Journal of Classification 2012;29:91-116.

27. Lê S, Josse J, Husson F (2008) FactoMineR: An R Package for Multivariate Analysis Journal of Statistical Software 2008;25:1-18.

28. Whitty CJM, MacEwen C, Goddard A, Alderson D, Marshall M, Calderwood C, Atherton F, McBride M, Atherton J, Stokes-Lampard H, Reid W, Powis S, Marx C Rising to the challenge of multimorbidity. BMJ 2020;368:16964.

29. Jønsson AR, Reventlow S, Guassora AD. How Older Men With Multimorbidity Relate to Successful Aging. J Gerontol B Psychol Sci Soc Sci 2020;75:1104-1112.

30. Chaudhry SI, McAvay G, Ning Y, Allore HG, Newman AB, Gill TM. Geriatric impairments and disability: the cardiovascular health study. J Am Geriatr Soc 2010;58:1686-92.

31. Chaudhry SI, Wang Y, Gill TM, Krumholz HM. Geriatric conditions and subsequent mortality in older patients with heart failure. J Am Coll Cardiol 2010;55:309-16.

32. Salisbury C, Man MS, Bower P, Guthrie B, Chaplin K, Gaunt DM, Brookes S, Fitzpatrick B, Gardner C, Hollinghurst S, Lee V, McLeod J, Mann C, Moffat KR, Mercer SW. Management of multimorbidity using a patient-centred care model: a pragmatic cluster-randomised trial of the 3D approach. Lancet 2018;392:41-50.

33. Reeve BB, Hays RD, Bjorner JB, Cook KF, Crane PK, Teresi JA, Thissen D, Revicki DA, Weiss DJ, Hambleton RK, Liu H, Gershon R, Reise SP, Lai J, Cella D, PROMIS Cooperative Group. Psychometric evaluation and calibration of health-related quality of life item banks: plans for the Patient-Reported Outcomes Measurement Information System (PROMIS). Med Care 2007;45:S22-31.

34. Nguyen QD, Wu C, Odden MC, Kim DH. Multimorbidity Patterns, Frailty, and Survival in Community-Dwelling Older Adults. J Gerontol A Biol Sci Med Sci 2019;74:1265-1270.

How to cite this article: J.-F. Dartigues, K. Le Bourdonnec, M. Tabue-Teguo, et al. Co-Occurrence of Geriatric Syndromes and Diseases in the General Population: Assessment of the Dimensions of Aging. J Nutr Health Aging. 2022;26(1):37-45, http:// dx.doi.org/10.1007/s12603-021-1722-3 